\title{
Biological Defenses against Ultra-Violet, Visible Light, and Near-Infrared Exposure
}

\author{
Yohei Tanaka1, Hideki Motomura'2, Masafumi Jinno² \\ ${ }^{1}$ Clinica Tanaka Plastic, Reconstructive Surgery and Anti-aging Center, Matsumoto, Japan \\ ${ }^{2}$ Department of Electrical and Electronic Engineering, Ehime University, Matsuyama, Japan \\ Email: info@clinicatanaka.jp
}

Received 7 December 2015; accepted 24 January 2016; published 28 January 2016

Copyright (C) 2016 by authors and Scientific Research Publishing Inc.

This work is licensed under the Creative Commons Attribution International License (CC BY). http://creativecommons.org/licenses/by/4.0/

(c) (i) 0pen Access

\begin{abstract}
Objective: Humans are increasingly exposed to artificial light and electromagnetic wave radiation, in addition to solar radiation. Many studies have shown the biological effects of ultra-violet and near-infrared exposure, but few have extensively investigated the innate biological defenses within human tissues against visible light and near-infrared exposure. Herein, we investigated spectral properties of endogenous human biological defenses against ultra-violet to near-infrared. Methods: A double-beam spectrophotometer $(190-2700 \mathrm{~nm})$ was used to measure the transmission spectra of a saline solution used to imitate perspiration, and oil to imitate sebum, as well as human skin, blood, adipose tissue, and muscle. Results: Saline (thickness, $0.5 \mathrm{~mm}$ ) blocked $27.5 \%$ - 98.6\% of ultra-violet, $13.2 \%-34.3 \%$ of visible light, and $10.7 \%-99.8 \%$ of near-infrared. Oil (thickness, $0.5 \mathrm{~mm}$ ) blocked $50.5 \%-100 \%$ of ultra-violet, $28.7 \%-54.8 \%$ of visible light, and $19.0 \%-98.3 \%$ of near-infrared. Blood thicknesses of 0.05 and $0.5 \mathrm{~mm}$ blocked over $97.8 \%, 100 \%$ of ultra-violet, over $\mathbf{9 4 . 6 \%}, \mathbf{9 9 . 7 \%}$ of visible light, and over $75.8 \%, 99.4 \%$ of near-infrared, respectively. Skin thicknesses of 0.25 and $0.5 \mathrm{~mm}$ blocked over $99.4 \%, 100 \%$ of ultra-violet and over $94.3 \%, 99.7 \%$ of visible light, and over $74.7 \%, 93.5 \%$ of near-infrared, respectively. Adipose tissue thickness of 0.25 and $0.5 \mathrm{~mm}$ blocked over $98.3 \%, 100 \%$ of ultra-violet, over $94.7 \%, 99.7 \%$ of visible light, and over $88.1 \%, 98.6 \%$ of near-infrared, respectively. Muscle thickness of 0.25 and0.5 mm blocked over $95.4 \%, 99.8 \%$ of ultra-violet, over $93.1 \%, 99.5 \%$ of visible light, and over $86.9 \%$, $\mathbf{9 8 . 3 \%}$ of near-infrared, respectively. Conclusions: Humans possess endogenous biological protection against ultra-violet, visible light and near-infrared exposure on multiple levels, including through perspiration, sebum, blood, skin, adipose tissue, and muscle. Since solar and artificial radiation affects human tissues, biological defenses made of biological materials may be induced to protect subcutaneous tissues against these wavelengths.
\end{abstract}

\section{Keywords}

Biological Defenses, Near-Infrared, Ultra-Violet, Visible Light 


\section{Introduction}

Incident solar energy is composed of 6.8\% ultra-violet (UV), 38.9\% visible light (Vis), and 54.3\% near-infrared (NIR) [1]. Chronic light and electromagnetic wave exposure may induce various kinds of tissue damage and disease. When life first originated on Earth, the first life forms appeared in water and thus they did not need to adapt to dehydration, gravity, or sunlight including UV, Vis, and NIR as the surrounding water served as a barrier against these obstacles [2]. The necessity to overcome these obstacles first arose when primitive life developed on land [2]. All species of terrestrial life have acquired fibrous structural proteins rich in hydrogen bonds and alpha helices: cellulose for plants, fibrous chitin and proteins for insects, and keratin for reptiles, birds, amphibians, and mammals [2].

The fibrous structural proteins rich in hydrogen bonds and alpha helices are particularly useful for blocking NIR because they efficiently absorb these wavelengths [2]. For instance, insects can remain in sunny environments without much perspiration because they consist of fibrous chitin and alpha helical proteins that efficiently block and absorb NIR, thereby preventing their body temperature from becoming elevated. Likewise, sheep prefer the sun to the shade, and even though these animals remain in sunny environments without much perspiration, their body temperature does not become elevated as sheep wool consists of alpha helical structures [2]. Humans possess endogenous protective mechanisms against UV, Vis, and NIR exposure on multiple levels, including perspiration, blisters, sebum, hair, melanin, vasodilation, skin, and adipose tissue, in addition to protective materials such as cotton or wool clothing [2]. Although many studies have shown the defensive capabilities of human tissues against UV exposure, their ability to protect against Vis and NIR exposure has not been thoroughly investigated.

Despite the prevalence of a variety of UV blocking materials, such as sunblock for skin, eyewear, clothing fibers, and films for glasses, monitors, personal computers, cars, and buildings, many of these items do not block Vis and/or NIR [2]. Previous NIR studies have described its application in industrial and agricultural fields. In medicine, the biological effects of sun and UV exposure have been extensively investigated. Although $40 \%$ of solar energy is Vis, and over half is NIR, the defensive capabilities of human tissues against Vis and NIR are not well understood. We have previously reported that NIR can induce various biological effects [2]-[21], including undesirable photoaging [2] [12] [14]-[17] [19] [21], sustained vasodilation [10], muscle thinning [6] [8], skin sagging and ptosis [14]-[17] [19], and potentially photocarcinogenesis.

Hence, further protection against the biological effects of Vis and NIR should be considered. This study investigated endogenous human defenses against UV, Vis, and NIR from biological perspective.

\section{Materials and Methods}

Saline (154 mEq/L; Otsuka Pharmaceutical Factory, Inc., Tokushima, Japan) was used to imitate perspiration, while jojoba oil was used to imitate sebum in the study. Skin, blood, adipose tissue, and muscle were taken from the corresponding author (age, 39 years-old; Japanese) after an operation to remove pigmented nevus at the Clinica Tanaka Anti-Aging Center (Nagano, Japan). When the pigmented nevus was removed by elliptical excision, excess skin on either side of the nevus was submitted for biopsy, and a very small amount of blood, adipose tissue and muscle were obtained. He had no history of any type of disease or medical treatment that could have affected biopsy results.

A double-beam spectrophotometer V-670 (Jasco Corp., Tokyo, Japan) was used to measure the transmission spectra. The spectrophotometer utilizes a unique, single monochromatic design covering a wavelength range of 190 to $2700 \mathrm{~nm}$ with a $+/-0.3 \mathrm{~nm}$ wavelength accuracy (UV/Vis bandwidth: $0.5 \mathrm{~nm}$ stabilized at room temperature), $+/-0.05 \mathrm{~nm}$ wavelength repeatability (UV-Vis bandwidth: $0.5 \mathrm{~nm}$ ), $+/-0.003$ photometric accuracy, $0.5-1$ absorbance, and $+/-0.3 \%$ transmittance (tested with NIST SRM 930D). The photometric range included 0 $10,000 \%$ transmittance with an absorbance of -2 to 4 in the UV/Vis region; emitted light was detected by a photomultiplier tube (PMT).

Figure 1 shows a simplified schematic diagram of the transmittance measurement. The light source (deuterium lamp, 190 - $340 \mathrm{~nm}$; tungsten lamp, 340 - $2700 \mathrm{~nm}$ ) emits UV, Vis and IR beams. The light was monochromated and irradiated the sample in the cuvette with an initial intensity of $I_{0}(\lambda)$ ). After absorption by the sample, the new light intensity $[I(\lambda)]$ was detected and measured by the PMT. The intensity before absorption was measured by placing the cuvette without sample in the spectrophotometer. Background noise $\left[I_{\mathrm{B}}(\lambda)\right]$ was measured by blocking the output beam from the monochromator. Finally, the transmittance of the sample, $T(\lambda)$,was 
determined as,

$$
T(\lambda)=\frac{I(\lambda)-I_{\mathrm{B}}(\lambda)}{I_{0}(\lambda)-I_{\mathrm{B}}(\lambda)} .
$$

The transmittance spectrum was obtained by rotating the grating in the monochromator to change the wavelength.

\section{Results}

The results of the transmission spectra of saline imitating perspiration, oil imitating sebum, skin, blood, adipose tissue, and muscle are shown in Figures 2(a)-(c), and the UV, Vis, and NIR blocking ability of each material are shown in Table 1.

Saline with a thickness of $0.5 \mathrm{~mm}$ blocked $41.5 \%$ - 98.6\% of UV-C (200 - $280 \mathrm{~nm}), 30.3 \%-41.5 \%$ of UV-B (280 - $315 \mathrm{~nm}$ ), 27.5\% - 30.3\% of UV-A (315 - $380 \mathrm{~nm}), 13.2 \%-34.3 \%$ of Vis (380 - $760 \mathrm{~nm}$ ), and 10.7\% 99.8\% of NIR. Oil with a thickness of $0.5 \mathrm{~mm}$ blocked $85.3 \%$ - 100\% of UV-C, $62.4-85.3 \%$ of UV-B, 50.5\% 62.4\% of UV-A, 28.7\% - 54.8\% of Vis, and 19.0\% - 98.3\% of NIR, as shown in Figure 2(a).

Blood with a thickness of 0.05 mm blocked $100 \%$ of UV-C, 99.5\% - 100\% of UV-B, 97.8\% - 99.6\% of UV-A, $94.6 \%-100 \%$ of Vis, and $75.8 \%-99.4 \%$ of NIR. Blood with a thickness of $0.5 \mathrm{~mm}$ blocked $100 \%$ of UV-A, UV-B, UV-C, 99.7\% - 100\% of Vis, and 99.4\% - 100\% of NIR, as shown in Figure 2(b).

Skin with a thickness of $0.25 \mathrm{~mm}$ blocked $100 \%$ of UV-C, $99.8 \%-100 \%$ of UV-B, $99.4 \%-99.8 \%$ of UV-A, $94.3 \%-99.4 \%$ of Vis, and $74.7 \%-99.9 \%$ of NIR. Skin with a thickness of $0.5 \mathrm{~mm}$ blocked $100 \%$ of UV-A, -B, and $-\mathrm{C}, 99.7 \%-100 \%$ of Vis, and 93.5\% - 100\% of NIR, as shown in Figure 2(c).

Adipose tissue with a thickness of $0.25 \mathrm{~mm}$ blocked 99.6\% - 100\% of UV-C, 98.9\% - 99.6\% of UV-B, 98.3\%

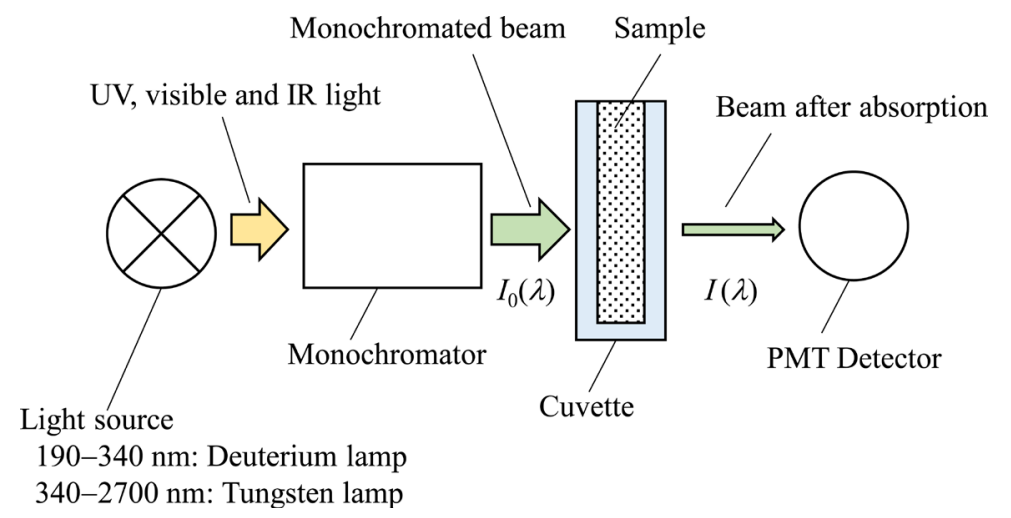

Figure 1. Simplified schematic diagram for transmittance measurements.

Table 1. Blocking abilities of saline, oil, skin, blood, adipose tissue, and muscle.

\begin{tabular}{|c|c|c|c|c|c|}
\hline & \multicolumn{5}{|c|}{ Blocking abilities } \\
\hline & UV-C & UV-B & UV-A & Vis & NIR \\
\hline Saline $0.5 \mathrm{~mm}$ & $41.5 \%-98.6 \%$ & $30.3 \%-41.5 \%$ & $27.5 \%-30.3 \%$ & $13.2 \%-34.3 \%$ & $10.7 \%-99.8 \%$ \\
\hline Oil $0.5 \mathrm{~mm}$ & $85.3 \%-100 \%$ & $62.4 \%-85.3 \%$ & $50.5 \%-62.4 \%$ & $28.7 \%-54.8 \%$ & $19.0 \%-98.3 \%$ \\
\hline Blood $0.05 \mathrm{~mm}$ & $100 \%$ & $99.5 \%-100 \%$ & $97.8 \%-99.6 \%$ & $94.6 \%-100 \%$ & $75.8 \%-99.4 \%$ \\
\hline Blood $0.5 \mathrm{~mm}$ & $100 \%$ & $100 \%$ & $100 \%$ & $99.7 \%-100 \%$ & $99.4 \%-100 \%$ \\
\hline Skin $0.25 \mathrm{~mm}$ & $100 \%$ & $99.8 \%-100 \%$ & $99.4 \%-99.8 \%$ & $94.3 \%-99.4 \%$ & $74.7 \%-99.9 \%$ \\
\hline Skin $0.5 \mathrm{~mm}$ & $100 \%$ & $100 \%$ & $100 \%$ & $99.7 \%-100 \%$ & $93.5 \%-100 \%$ \\
\hline Adipose tissue $0.25 \mathrm{~mm}$ & $99.6 \%-100 \%$ & $98.9 \%-99.6 \%$ & $98.3 \%-98.9 \%$ & $94.7 \%-98.3 \%$ & $88.1 \%-98.2 \%$ \\
\hline Adipose tissue $0.5 \mathrm{~mm}$ & $100 \%$ & $100 \%$ & $100 \%$ & $99.7 \%-100 \%$ & $98.6 \%-100 \%$ \\
\hline Muscle 0.25 mm & $96.3 \%-97.3 \%$ & $96.1 \%-96.3 \%$ & $95.4 \%-96.1 \%$ & $93.1 \%-95.5 \%$ & $86.9 \%-93.4 \%$ \\
\hline Muscle $0.5 \mathrm{~mm}$ & $99.9 \%-100 \%$ & $99.8 \%-99.9 \%$ & $99.8 \%-99.9 \%$ & $99.5 \%-99.8 \%$ & $98.3 \%-99.5 \%$ \\
\hline
\end{tabular}




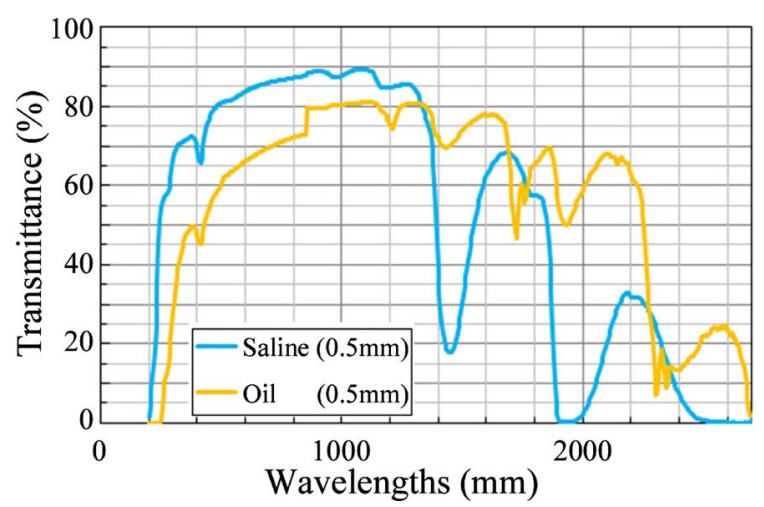

(a)

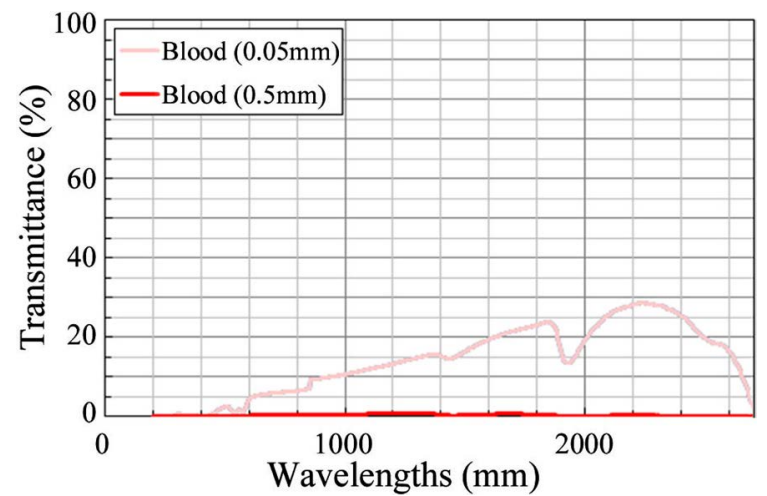

(b)

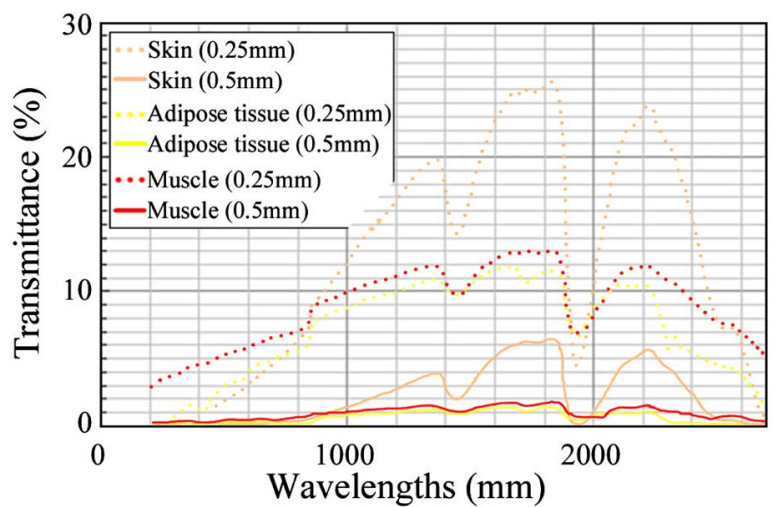

(c)

Figure 2. (a) A schematic of saline and oil transmittance; (b) A schematic of blood transmittance; (c) A schematic of tissue transmittance.

- 98.9\% of UV-A, 94.7\% - 98.3\% of Vis, and 88.1\% - 98.2\% of NIR. Adipose tissue with a thickness of $0.5 \mathrm{~mm}$ blocked $100 \%$ of UV-A,-B, and-C, 99.7\% - 100\% of Vis, and 98.6\% - 100\% of NIR, shown in Figure 2(c).

Muscle with a thickness of 0.25 mm blocked $96.3 \%-97.3 \%$ of UV-C, $96.1 \%-96.3 \%$ of UV-B, 95.4\% 96.1\% of UV-A, 93.1\% - 95.5\% of Vis, and 86.9\% - 93.4\% of NIR. Muscle with a thickness of $0.5 \mathrm{~mm}$ blocked $99.9 \%-100 \%$ of UV-C, $99.8 \%$ - 99.9\% of UV-B, and -A, 99.5\% - 99.8\% of Vis, and 98.3\% - 99.5\% of NIR, as shown in Figure 2(c).

\section{Discussion}

In the current study, we demonstrated slight UV attenuation by saline imitating perspiration and oil imitating sebum on the skin and almost complete impedance by human blood and skin. Epidermal thickness and melanization 
are important factors for UV-C and UV-B, whereas the attenuation of UV-A is primarily via melanin [22].

Saline with a thickness of $0.5 \mathrm{~mm}$ blocked over $40 \%$ of UV-C, but could not sufficiently block UV-B and -A. Oil with a thickness of $0.5 \mathrm{~mm}$ blocked over $85 \%$ of UV-C, and 50\% of UV-B and -A. These results suggest that perspiration and/or sebum on the skin alone cannot sufficiently block most wavelengths of UV.

However, human skin itself appeared to be enough to mitigate UV because both 0.25 - and 0.5 -mm skin thicknesses were able to block over $99 \%$ of all UV wavelengths tested. This is notable since the thinnest piece of human skin regularly exposed to the sun is the eyelid (thickness, $\geq 0.5 \mathrm{~mm}$ ).

While Vis was only slightly attenuated by saline and oil, it was almost completely blocked (>90\%) by human skin, blood, adipose tissue, and muscle. Wavelengths below $650 \mathrm{~nm}$ are absorbed by hemoglobin in the skin, while wavelengths below $1100 \mathrm{~nm}$ are absorbed by melanin [23]. Blood with a thickness of $0.05 \mathrm{~mm}$ and skin with a thickness of $0.25 \mathrm{~mm}$ blocked over $94 \%$ of Vis, while blood and skin with a thickness of $0.5 \mathrm{~mm}$ blocked $99.7 \%-100 \%$ of Vis. These data suggest that even the thinnest areas of human blood and skin are enough to block Vis.

NIR was also only slightly attenuated by saline and oil, and blocked (>70\%) by skin, blood, adipose tissue, and muscle. NIR wavelengths between 1400 and $1500 \mathrm{~nm}$ are absorbed by hemoglobin and water in the skin, while wavelengths above $1850 \mathrm{~nm}$ are absorbed by water therein [23]. Absorption peaks in water transmission spectra are related to the $\mathrm{O}-\mathrm{H}$ bond in water molecules [24], and the NIR spectrum of biological materials basically results from overtones and combinations of $\mathrm{O}-\mathrm{H}, \mathrm{C}-\mathrm{H}$, and $\mathrm{N}-\mathrm{H}$ bond stretching [25]. Since water and fatty acids are the major components of skin and consist of $\mathrm{O}-\mathrm{H}$ and $\mathrm{C}-\mathrm{H}$ intramolecular bonds, these molecules are the major NIR-absorbing materials in soft tissues [24].

Whereas skin with a thickness of $0.25 \mathrm{~mm}$ blocked over $74.7 \%$ of NIR, skin with a thickness of $0.5 \mathrm{~mm}$ blocked over 93.5\%. These results indicate that skin thickness is important for NIR protection; thin skin likely allows deeper penetration of UV, Vis, and NIR wavelengths, allowing deeper tissue damage as opposed to thicker skin.

Interestingly, even a small amount of blood (thickness, $0.05 \mathrm{~mm}$ ) blocked over $75.8 \%$ of NIR, being roughly comparable to 0.25 -mmthick skin. Blood with a thickness of $0.5 \mathrm{~mm}$ blocked $100 \%$ of UV, $99.7 \%-100 \%$ of Vis, over $99.4 \%$ of NIR. These results indicate that blood is an effective sunscreen. Furthermore, if skin is thin, UV, Vis, and NIR will induce vasodilation to protect subcutaneous tissues.

NIR is thought to mainly resonate with hydrogen bonds, and alpha helices [26]. Both hemoglobin and myoglobin are oxygen-carrying proteins comprised many alpha helices. It is possible that NIR induces resonance of helical structures in these and other proteins resulting in their degeneration, which would damage important oxygen storage and transport mechanisms in the body, lead to cellular apoptosis and eventually persistent vasodilation [2] [12].

For example, rosacea can be induced by NIR exposure and is more common in Caucasians and fair-skinned populations [27]. Degeneration of myoglobin and apoptosis of vascular smooth muscle cells induced by NIR results in sustained vasodilation [10], which protects against NIR exposure by increasing water and hemoglobin retention [10].

Adipose tissue with a thickness of 0.25 and $0.5 \mathrm{~mm}$ blocked over $94.7 \%$ and $99.7 \%$ of Vis and over $88.4,98.6 \%$ of NIR, respectively. The blocking ability of adipose tissue was much higher than those of oil, even though both contain fatty acids. However, adipose tissue also contains blood vessels, which may play an important role in enhancing the blocking ability of this tissue. Long-term exposure to NIR induces subcutaneous adipocytes located above superficial muscles which can effectivelyscatter and block NIR [9].

Muscle with a thickness of 0.25 and $0.5 \mathrm{~mm}$ blocked over 93.1 and $99.5 \%$ of Vis, and over $86.9,98.3 \%$ of NIR, respectively. Muscles are easily damaged by NIR, because they contain hemoglobin and myoglobin [8] [12]. Therefore, muscle is protected by skin and subcutaneous tissues. Superficial muscle thinning is induced by chronic NIR exposure, which ultimately leads to superficial tissue aging and skin ptosis [14] [19]. Fair skin tends to wrinkle and sag earlier in life [28] [29], and characteristic age-related skin changes occur at a more accelerated rate in Caucasians [30]. Thus, patients seeking facelifts often have fair skin and/or a habit of sunbathing. Chronic sunbathing induces undesirable photoaging, sustained vasodilation, muscle thinning, skin ptosisand laxity, cataracts, and oncogenicity.

Our results showed that skin and adipose tissue with a thickness of $0.5 \mathrm{~mm}$ can sufficiently protect the underlying superficial muscles from UV, Vis, and NIR exposure. Thus, humans possess endogenous protection against UV, Vis, and NIRwavelengths on multiple levels, including perspiration, sebum, blood, skin, adipose 
tissue, and muscle. Interestingly, although humans perspire to increase superficial water retention and thereby decrease superficial temperature, subsequent vasodilation to increase water and hemoglobin retention is NIR absorbent.

In the dermis, NIR induces accumulation of collagen, elastin and water-binding proteins, which possess alpha helical structures rich in hydrogen bonds [2]. Not only do humans artificially protect themselves from NIR deep tissue damage by wearing protective materials (e.g., cotton or wool clothing) rich in hydrogen bonds and alpha helices, the water in their perspiration and blisters, sebum secretions, keratin in hair, the stratum corneum, hemoglobin, water, and water-binding proteins in their blood and dermis, and underlying adipose tissue all serve to reflect/absorb NIR. Hence, treatments which remove hair and the stratum corneum, such as laser hair removal, and chemical and laser peeling, decrease the endogenous solar-protective ability of these structures.

Furthermore, more sensitive and exposed areas like the eyes and optic nerves are protected from NIR by keratin, water-binding proteins, and fatty acid in the eyelids, keratin in eyelashes, tears in sclera, as well as water and hyaluronic acid in the lens and blood flow in the retina. All of these naturally occurring, biological components of the human body are ideal solar-protective materialsrich in hydrogen bonds and alpha helices which block NIR exposureand subsequent tissue damage [2].

\section{Conclusion}

Saline used to imitate perspiration, oil to imitate sebum secretions, as well as skin, blood, adipose tissue, and muscle can attenuate or block light and electromagnetic wave ranging in wavelengths from 190 to $2700 \mathrm{~nm}$. Individuals with thin skin or those removing sebum, keratin, and/or stratum corneum and/or exposing their skin and eyes should protect their tissues by using protective materials to prevent the deleterious effects of light and electromagnetic wave.

\section{References}

[1] Kochevar, I.E., Pathak, M.A. and Parrish, J.A. (1999) Photophysics, Photochemistry and Photobiology. In: Freedberg, I.M., Eisen, A.Z., Wolff, K., et al., Eds., Fitzpatrick’s Dermatology in General Medicine, McGraw-Hill, New York, 220-229.

[2] Tanaka, Y. and Gale, L. (2013) Beneficial Applications and Deleterious Effects of Near-Infrared from Biological and Medical Points of View. Optics and Photonics Journal, 3, 31-39. http://dx.doi.org/10.4236/opj.2013.34A006

[3] Tanaka, Y., Matsuo, K., Yuzuriha, S. and Shinohara, H. (2009) Differential Long-Term Stimulation of Type I versus Type III Collagen after Infrared Irradiation. Dermatologic Surgery, 35, 1099-1104. http://dx.doi.org/10.1111/j.1524-4725.2009.01194.X

[4] Tanaka, Y., Matsuo, K. and Yuzuriha, S. (2009) Long-Term Evaluation of Collagen and Elastin Following Infrared (1100 to $1800 \mathrm{~nm}$ ) Irradiation. J Drugs Dermatol, 8, 708-712.

[5] Tanaka, Y., Matsuo, K., Yuzuriha, S., Yan, H.M. and Nakayama, J. (2010) Non-Thermal Cytocidal Effect of Infrared Irradiation on Cultured Cancer Cells Using Specialized Device. Cancer Science, 101, 1396-1402. http://dx.doi.org/10.1111/j.1349-7006.2010.01548.x http://onlinelibrary.wiley.com/doi/10.1111/j.1349-7006.2010.01548.x/epdf

[6] Tanaka, Y., Matsuo, K. and Yuzuriha, S. (2010) Long-Lasting Muscle Thinning Induced by Infrared Irradiation Specialized with Wavelength and Contact Cooling: A Preliminary Report. ePlasty, 10, e40. http://www.eplasty.com/index.php?option=com_content\&view=article\&id=453\&catid=171:volume-10-eplasty-2010

[7] Tanaka, Y., Matsuo, K. and Yuzuriha, S. (2010) Long-Term Histological Comparison between Near-Infrared Irradiated Skin and Scar Tissues. Clinical, Cosmetic and Investigational Dermatology, 3, 143-149. http://dx.doi.org/10.2147/CCID.S15729 http://www.dovepress.com/articles.php?article_id=5752

[8] Tanaka, Y., Matsuo, K. and Yuzuriha, S. (2011) Long-Lasting Relaxation of Corrugator Supercilii Muscle Contraction Induced by Near Infrared Irradiation. ePlasty, 11, e6. http://www.eplasty.com/index.php?option=com_content\&view=article\&id=519\&catid=172:volume-11-eplasty-2011

[9] Tanaka, Y., Matsuo, K. and Yuzuriha, S. (2011) Near-Infrared Irradiation Non-Thermally Affects Subcutaneous Adipocytes and Bones. ePlasty, 11, e12. http://www.eplasty.com/index.php?option=com_content\&view=article\&id=528\&catid=172:volume-11-eplasty-2011

[10] Tanaka, Y., Matsuo, K. and Yuzuriha, S. (2011) Near-Infrared Irradiation Non-Thermally Induces Long-Lasting Vasodilation by Causing Apoptosis of Vascular Smooth Muscle Cells. ePlasty, 11, e22. http://www.eplasty.com/index.php?option=com_content\&view=article\&id=541\&catid=172:volume-11-eplasty-2011 
[11] Tanaka, Y., Matsuo, K. and Yuzuriha, S. (2011) Objective Assessment of Skin Rejuvenation Using Near-Infrared 1064-nm Neodymium: YAG Laser in Asians. Clinical, Cosmetic and Investigational Dermatology, 4, 123-130. http://dx.doi.org/10.2147/CCID.S22841

[12] Tanaka, Y. and Matsuo, K. (2011) Non-Thermal Effects of Near-Infrared Irradiation on Melanoma. In: Tanaka, Y., Ed., Breakthroughs in Melanoma Research, InTech, Croatia, 597-628. http://dx.doi.org/10.5772/38663 http://www.intechopen.com/books/breakthroughs-in-melanoma-research/non-thermal-effects-of-near-infrared-irradiati on-on-melanoma

[13] Tanaka, Y., Tatewaki, N., Nishida, H., Eitsuka, T., Ikekawa, N. and Nakayama, J. (2012) Non-Thermal DNA Damage of Cancer Cells Using Near-Infrared Irradiation. Cancer Science, 103, 1467-1473. http://dx.doi.org/10.1111/j.1349-7006.2012.02310.x http://onlinelibrary.wiley.com/doi/10.1111/j.1349-7006.2012.02310.x/epdf

[14] Tanaka, Y. (2012) Impact of Near-Infrared in Dermatology. Review. World Journal of Gastroenterology, 1, 30-37. http://dx.doi.org/10.5314/wjd.v1.i3.30

[15] Tanaka, Y. and Kawashima, M. (2012) The Biological Effects of Near-Infrared. Aesthetic Dermatology, 22, $100-109$. (In Japanese)

[16] Tanaka, Y., Tunemi, Y., Kawashima, M. and Nishida, H. (2013) The Impact of Near-Infrared in Plastic Surgery. Plastic Surgery: An International Journal, 2013, Article ID: 973073. http://dx.doi.org/10.5171/2013.973073 http://www.ibimapublishing.com/journals/PSIJ/2013/973073/973073.pdf

[17] Tanaka, Y. and Gale, L. (2013) The Effect of Near-Infrared between 1100-1800 nm Together with a Water-Filter and a Contact Cooling. Anaplastology, 2, 111.

[18] Tanaka, Y., Tunemi, Y., Kawashima, M., Tatewaki, N. and Nishida, H. (2013) Objective Assessment of Skin Tightening Using Water-Filtered Near-Infrared (1000-1800 nm) Device with a Contact Cooling and Freezer Stored Gel in Asians. Clinical, Cosmetic and Investigational Dermatology, 6, 167-176. http://dx.doi.org/10.2147/CCID.S47299

[19] Tanaka, Y. and Gale, L. (2013) The Necessity of Near-Infrared Protection. Surgery: Current Research, 3, 150.

[20] Tanaka, Y., Tatewaki, N., Fujimoto, T. and Nishida, H. (2014) Non-Thermal Anticancer Effects of Water-Filtered Broad-Spectrum Near-Infrared Irradiation. International Journal of Cancer Research, 48, 1258-1265.

[21] Tanaka, Y. and Gale, L. (2015) Protection Fromnear-Infrared to Prevent Skin Damage. Optics and Photonics Journal, 5, 113-118. http://dx.doi.org/10.4236/opj.2015.54010

[22] Anderson, R.R. and Parrish, J.A. (1981) The Optics of Human Skin. Journal of Investigative Dermatology, 77, 13-19. http://dx.doi.org/10.1111/1523-1747.ep12479191

[23] Kelleher, D.K., Thews, O., Rzeznik, J., Scherz, A., Salomon, Y. and Vaupel, P. (1999) Hot Topic. Water-Filtered Infrared-A Radiation: A Novel Technique for Localized Hyperthermia in Combination with Bacteriochlorophyll-Based Photodynamic Therapy. International Journal of Hyperthermia, 15, 467-474. http://dx.doi.org/10.1080/026567399285468

[24] Tsai, C.H., Chen, J.C. and Wang, W.J. (2001) Near-Infrared Absorption Property of Biological Soft Tissue Constituents. Journal of Medical and Biological Engineering, 21, 7-14.

[25] Weyer, L.G. (1985) Near-Infrared Spectroscopy of Organic Substances. Applied Spectroscopy Reviews, 21, 1-43. http://dx.doi.org/10.1080/05704928508060427 http://www.tandfonline.com/doi/abs/10.1080/05704928508060427\#.VQO8dpSCh0I

[26] Nevskaya, N.A. and Chirgadze, Y.N. (1976) Infrared Spectra and Resonance Interactions of Amide-I and II Vibrations of Alpha-Helix. Biopolymers, 15, 637-648. http://dx.doi.org/10.1002/bip.1976.360150404

[27] Berg, M. (1989) Epidemiological Studies of Influence of Sunlight on the Skin. Photodermatology, 6, 80-84.

[28] Rawlings, A.V. (2006) Ethnic Skin Types: Are There Differences in Skin Structure and Function? Review Article. International Journal of Cosmetic Science, 28, 79-93. http://dx.doi.org/10.1111/j.1467-2494.2006.00302.x

[29] Tsukahara, T., Fujimura, T., Yoshida, Y., Kitahara, T., Hotta, M., Moriwaki, S., Witt, P.S., Simion, F.A. and Takema, Y. (2004) Comparison of Age-Related Changes in Wrinkling and Sagging of the Skin in Caucasian Females and in Japanese Females. International Journal of Cosmetic Science, 55, 314. http://dx.doi.org/10.1111/j.1467-2494.2004.00245_5.x

[30] Odunze, M., Rosenberg, D.S. and Few, J.W. (2008) Periorbital Aging and Ethnic Considerations: A Focus on Leteralcanthal Complex. Plastic \& Reconstructive Surgery, 121, 1002-1008. http://dx.doi.org/10.1097/01.prs.0000299381.40232.79 